\title{
Noble gas incorporation into silicate glasses: implications for planetary volatile storage
}

\author{
H. Yang ${ }^{1 *}$, A.E. Gleason ${ }^{1,2}$, S.N. Tkachev ${ }^{3}$, B. Chen ${ }^{4}$, \\ R. Jeanloz ${ }^{5}$, W.L. Mao ${ }^{1,2}$
}

Abstract

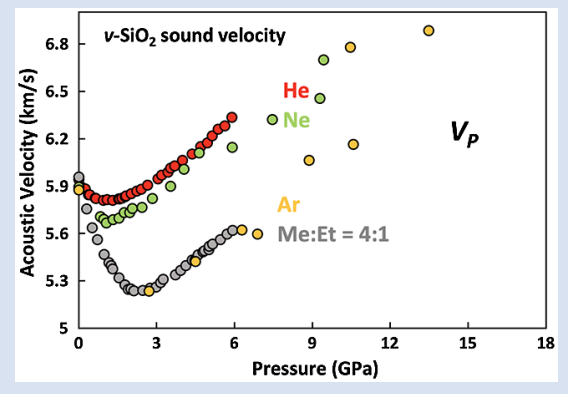

Incorporation of small molecules in silicate melts may provide an important mechanism for storing noble gases in the deep Earth, yet the means by which chemically inert noble gases enter and are retained in silica-based materials is not understood. High pressure, room temperature sound velocity measurements on silica and natural basalt glasses in different pressure-transmitting media reveal that neon enters the structure of silicate glasses and enhances their elastic strengths, whereas an ethanol-methanol mixture does not. Combined with literature data, we found the incorporation of small molecules into silica and basalt glasses is controlled by the void size distribution of the glass and size of the molecules. Pressure primarily reduces the size of noble gases, thereby increasing their solubilities in silicate melts and glasses.

Received 21 September 2020 | Accepted 6 January 2021 | Published 10 February 2021

\section{Introduction}

Radiogenic heat production generates ${ }^{40} \mathrm{Ar},{ }^{21} \mathrm{Ne}$ and ${ }^{4} \mathrm{He}$ inside Earth, and the ratios of these isotopes to non-radiogenic isotopes have been used to infer the style of mantle convection and the source of ocean island basalts (e.g., Mukhopadhyay and Parai, 2019). Owing to their changing reactivity and volatility with pressure, noble gases are also useful geochemical tracers for the interior processes of planets (e.g., Sanloup et al., 2005). However, how these noble gases are distributed among potential geochemical reservoirs, and how they alter the physical properties of their host with increasing depth (and therefore pressure) is still unclear. The storage of noble gases in quartz, ferropericlase, and bridgmanite at high pressure has been experimentally verified (Sanloup et al., 2005; Rosa et al., 2020). Nevertheless, the partition coefficients of noble gases between minerals and melts are on the order of $10^{-3}$ (Karato, 2016), implying significant storage of noble gases in silicate melts. This deep storage could also potentially alter atmospheric composition. As silicate glasses and melts share structural similarities (Williams and Jeanloz, 1988; Morard et al., 2020), with glass being the kinetically hindered state of the corresponding melt, understanding the incorporation of noble gases into silicate glasses can shed light on their storage in natural silicate melts.

Noble gases are widely used as pressure-transmitting media in high pressure diamond anvil cell experiments. These gases are chemically inactive and display relatively low mechanical strength, and thus minimise pressure gradients and deviatoric stresses in the sample chamber (Klotz et al., 2009). Use of noble gases as pressure-transmitting media presumes minimal interaction with the pressurised sample, yet there have been several reports that helium penetrates into the structure of silica glass at room temperature, enhancing both its incompressibility and rigidity (Sato et al., 2011; Shen et al., 2011; Weigel et al., 2012). Another study on basalt and enstatite glasses also indicates neon can enter their structure at high pressure (Clark et al., 2016).

Void space analysis of silica could shed light on the incorporation of noble gases into its structure. Theoretical simulations of the structure of silica glass and void size analysis have provided statistics on the interstitial space (i.e. the largest spherical site not occupied by $\mathrm{Si}$ or $\mathrm{O}$ ) that could potentially be available for incorporating noble gases (Shackelford and Masaryk, 1978; Malavasi et al., 2006). However, systematic study of the high pressure solubility of these gases in silica glass has been lacking, despite a number of studies on helium (Sato et al., 2011; Shen et al., 2011; Weigel et al., 2012).

To help clarify the mechanism of noble gas incorporation into amorphous silica and natural silicate glasses, we measured high pressure Brillouin spectra of silica and basalt glasses using different pressure-transmitting media at room temperature. The measured elastic properties of the material provide insight into the structural evolution and molecule incorporation of each glass

\footnotetext{
1. Department of Geological Sciences, Stanford University, Stanford, CA 94305, USA

. SLAC National Accelerator Laboratory, Menlo Park, CA 94025, USA

Center for Advanced Radiation Sources, University of Chicago, Chicago, Illinois 60637, USA

Center for High Pressure Science and Technology Advanced Research, Pudong, Shanghai 201203, China

Earth and Planetary Sciences Department, University of California, Berkeley, CA 94720, USA

Corresponding author (email: hyang666@stanford.edu)
} 
with compression. Elasticity is a useful monitor of the solution process because in situ measurement of gas solubility is challenging at high pressure. Together with existing literature data, we provide a comprehensive review of noble gases migrating into silica glass and natural basalt glasses under pressure. We find that solubility is controlled by the atomic sizes of the noble gases relative to the size of available interstitial spaces in the silicate glasses. Pressure alters both factors, thereby affecting the solubility of noble gases and other volatile species in glasses and melts.

\section{Results}

Details on sample synthesis, compositions, and data collection can be found in the Supplementary Information. We found that the sound velocities of silica glass depend on the pressuretransmitting medium (Fig. 1). We observed a drop in both compressional and shear velocities when increasing pressure between 1 and $3 \mathrm{GPa}$, followed by a slightly increasing or nearly unchanged velocity at higher pressures (Fig. 1). Silicate glasses with natural compositions share a similar framework structure with silica glass, and their velocity drop upon initial compression was also documented in other polymerised silicate glasses, such as basalt, jadeite and albite glasses (Liu and Lin, 2014; Sakamaki et al., 2014). In contrast, depolymerised glasses like diopside or enstatite glass do not show a decreasing trend, but rather an almost pressure-independent velocity (Sanchez-Valle and Bass, 2010; Liu and Lin, 2014; Sakamaki et al., 2014). These observations can be explained by the flexibility of $\mathrm{SiO}_{4}$ tetrahedra networks. In the low pressure range, below $3 \mathrm{GPa}$, the $\mathrm{SiO}_{4}$ tetrahedra in the glass rotate into the void space to form a high-density structure (Clark et al., 2016). The rotation does not involve substantial compression of the interatomic bonds, so the elastic moduli of the material remain largely unaltered. Therefore, the velocities, given by the square root of the ratio of the moduli and density, decrease during this stage. However, after the void space is filled, tetrahedral rotation is replaced by the interatomic bond shortening, and the sound velocities then increase under compression (Clark et al., 2016). Depolymerised silicate glasses, which contain larger 'modifier' cations like $\mathrm{Mg}^{2+}, \mathrm{Na}^{2+}$ or $\mathrm{Ca}^{2+}$, have less void space and

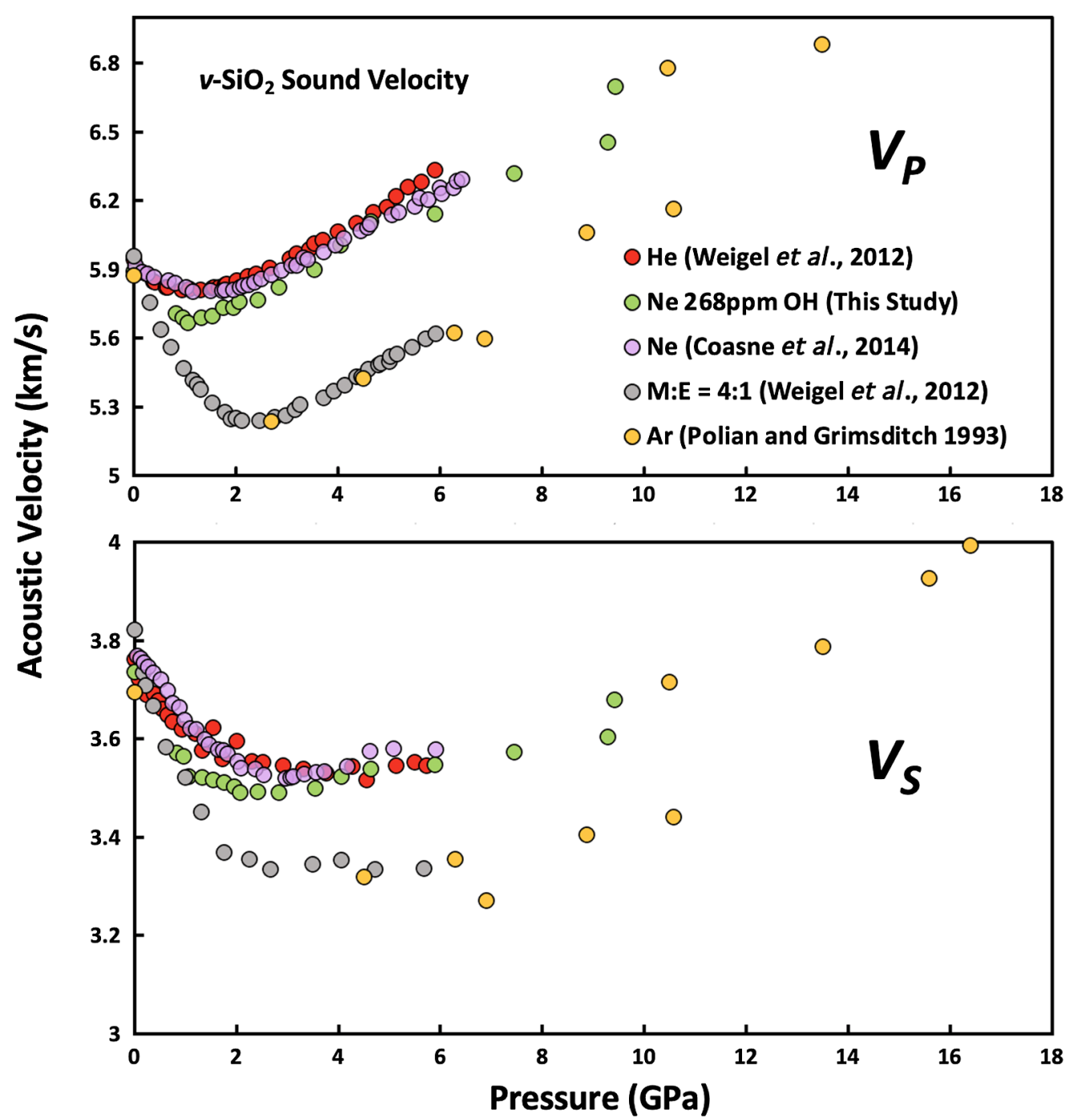

Figure 1 Sound velocities of vitreous silica under high pressure in different pressure media. M-E represents 4:1 Methanol-Ethanol mixture. Errors of the velocities are estimated from statistical uncertainties arising from the peak fitting. Typical errors are less than $1.5 \%$ and smaller than the size of the symbols. For both the $V_{P}$ and $V_{S}$ of silica in different noble gas media, we found a consistent trend for the acoustic velocities $-\mathrm{He}>\mathrm{Ne}>\mathrm{Ar} \approx \mathrm{M}-\mathrm{E}$. The abnormal velocity minimum at around 2-5 GPa can be attributed to the rearrangement of $\mathrm{SiO}_{4}$ tetrahedra in the vitreous silica structure (Clark et al., 2016). 


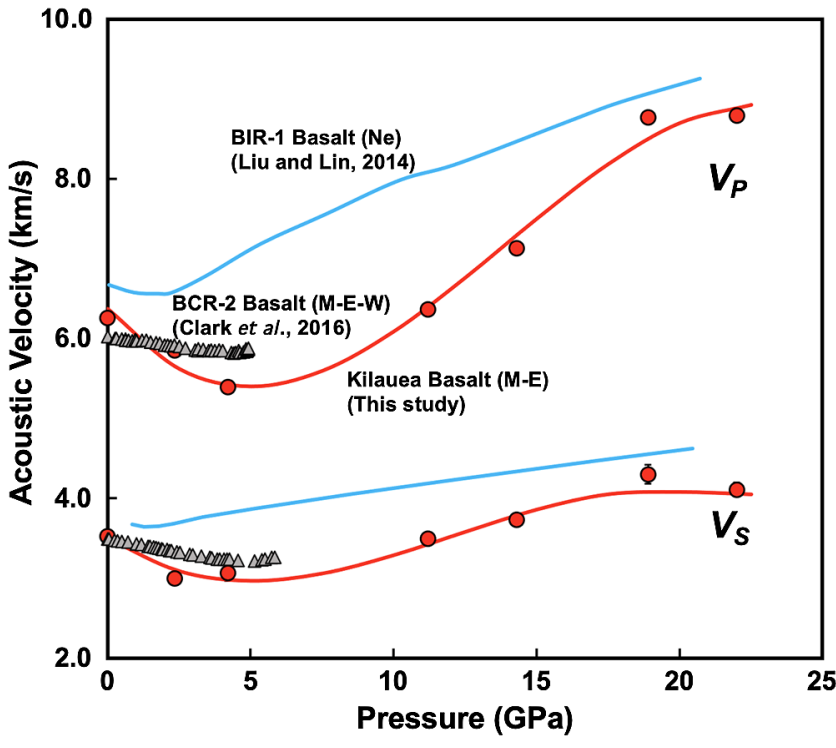

Figure 2 Sound velocities of basalt glasses at high pressure. M-E-W: 16:3:1 Methanol-Ethanol-Water mixture, M-E: 4:1 Methanol-Ethanol mixture. Errors of the velocities are estimated from statistical uncertainties arising from the peak fitting. Error bars smaller than the symbols plotted are not shown. Although the three basalt glasses have slightly different compositions, their degrees of polymerisation (NBO/T $=0.6,0.9$ and 0.8 for BCR-2, BIR1 and KB, respectively) are quite similar (BIR-1, blue coloured line, Liu and Lin, 2014; BCR-2, triangular points, Clark et al., 2016). The extents of the velocity drops are very different among the glasses. We observed that BIR-1 has a $2 \%$ drop for both $V_{P}$ and $V_{s}$, BCR-2 has a $2.8 \%$ drop for $V_{P}$ and a $7.2 \%$ drop for $V_{S}$, while KB in $\mathrm{M}-\mathrm{E}$ has a $14 \%$ drop for both $V_{P}$ and $V_{s}$. We attribute this variation to be mostly due to the different pressure media used. $\mathrm{H}_{2} \mathrm{O}$ and $\mathrm{Ne}$ have a relatively small molecule size that can possibly penetrate into the structure of silicate glass and make it stiffer (Figs. 3, S-4).

consequently less flexibility. The densification may also involve some chemical bond shortening and lead to the unchanged velocity profile with increasing pressure.

For basalt glasses, sound velocities at high pressure are also influenced by the pressure media (Fig. 2). The BIR-1 sample has higher velocities in a neon medium, as compared with basalt in M-E and M-E-W (methanol: ethanol: water = 16:3:1); it also has an earlier transition pressure at which the velocities start to increase. Comparing the M-E and M-E-W cases, we see that water seems to lower the decreasing slope below $5 \mathrm{GPa}$, but it does not change the transition point for the change in velocity trends (Fig. 2).

\section{Discussion}

Sound velocity data for amorphous materials can be very useful to calculate their density at high pressure (Zha et al., 1994). However, this method would fail if pressure media penetrates the sample (Weigel et al., 2012). We calculated the $P-V$ curve from velocities observed in different media and used this to examine whether incorporation of pressure media occurred in our experiments (see Supplementary Information for details). Neon and helium seem also able to penetrate into the silica structure while water and methanol molecules seem also to be able to penetrate into basalt glasses (Fig. S-3).

We compared the molecular size of the pressure media with the size of the interstitial space in the silica structure (Figs. 3, S-4). At ambient conditions, the sizes of helium, neon
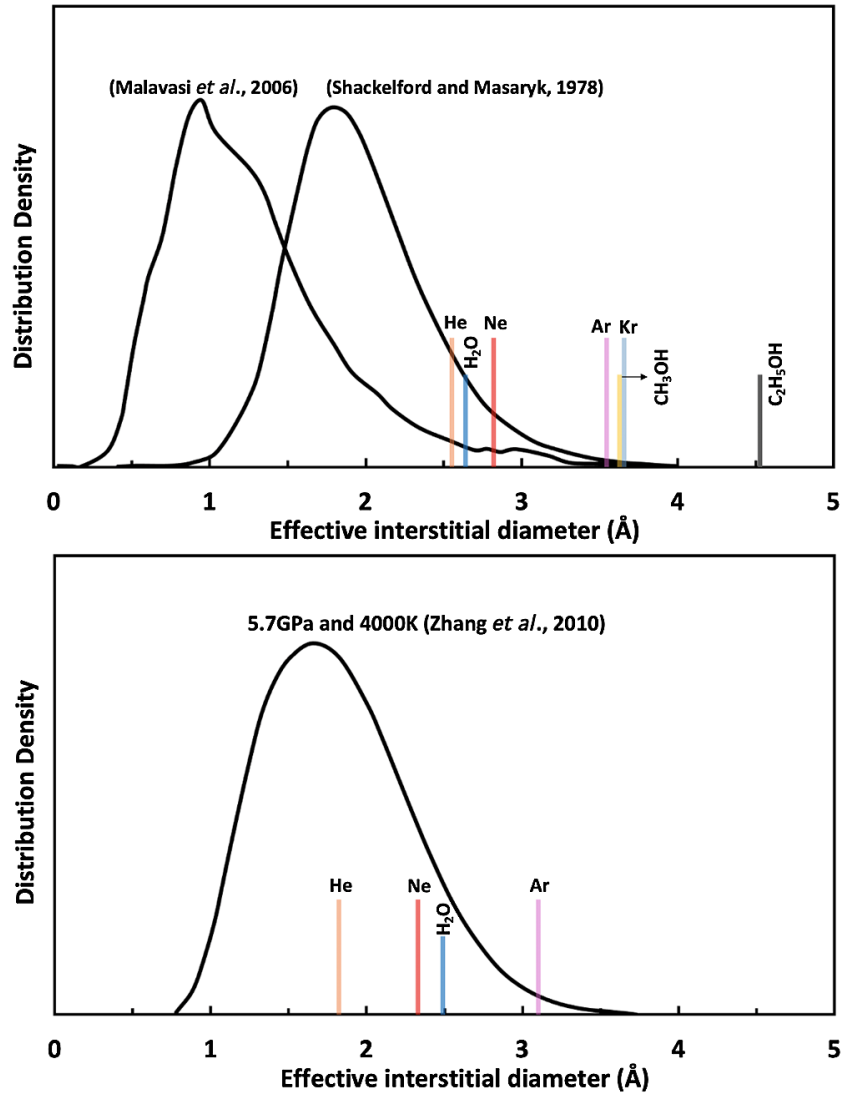

Figure 3 Void size distribution of $\mathrm{SiO}_{2}$ and molecular size of common pressure media at ambient condition and high pressure. Top panel: Molecular size data at ambient conditions were adapted from Reid et al. (1987). Bottom panel: High pressure molecule sizes were calculated using equations of state (He: Loubeyre et al., 1993; Ne: Dewaele et al., 2008; Ar: Ross et al., 1986, $\mathrm{H}_{2} \mathrm{O}$ : Yoshimura et al., 2006).

and water molecules are smaller than some voids in the silica structure. At higher pressures, the void size distribution generally shifts to a smaller volume, but the peak position moves only slightly and is still larger than $1.75 \AA$. On the other hand, the sizes of highly compressible gases decrease dramatically with increasing pressure, especially for helium and neon (Fig. S-4). These results indicate that helium and neon elevate the elastic stiffness of silica by supporting the structure in the void space, while molecules larger than argon are too big to be incorporated into silica and do not show this effect. Pressure makes these atoms smaller, enhancing the solubility of neon and helium into silica (Fig. 3). Although argon also becomes smaller at high pressure, it is still larger than most of the voids in silica. Its solubility is limited and does not influence the elastic properties significantly (Fig. 1).

Our measurements do not provide solubility values, but by comparing the gas and non-gas experiments we can make an estimate of this parameter (Sato et al., 2011) (Fig. S-3). In Figure 4, the upper limit is constrained by the maximum available space in the silica structure. This space is calculated as the volume difference between normal silica and the ultra-dense six-coordinated silica extropolated to lower pressure. On the other hand, the lower limit is given by the difference between gas and non-gas curves, assuming the 'expansion' should wholly or partly come from the volume of gas in the structure. Since the partial volume of a component in a mixture is smaller than the volume on its own (Bajgain et al., 2015), the real solubility should be higher than the lower limit here. 


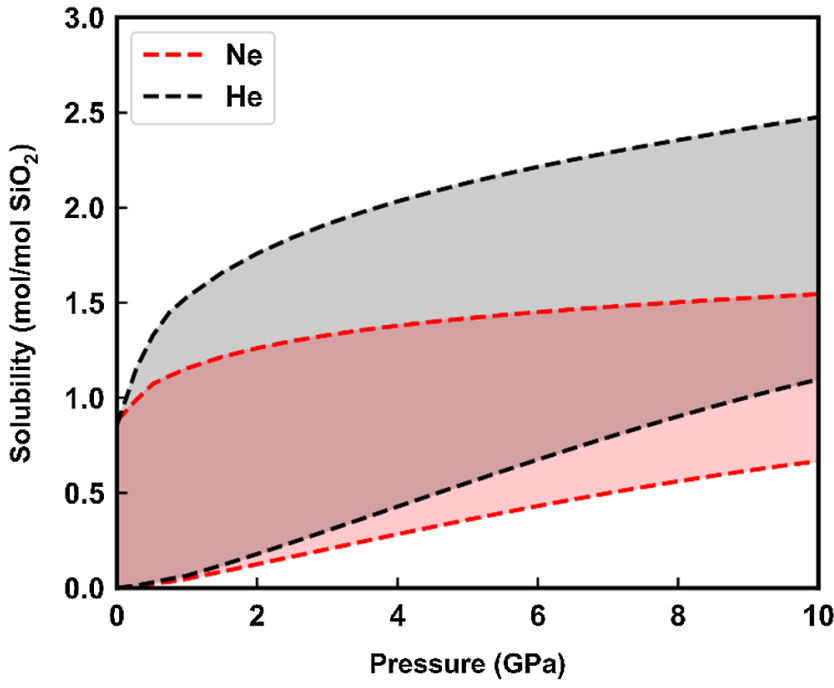

Figure 4 Solubility of neon and helium in vitreous silica at high pressure. The two lines for each medium represent upper limit and lower limits, respectively. The lower and upper limits were estimated by $\left(\mathrm{V}_{\text {rigid }}-\mathrm{V}_{\text {normal }}\right) / \mathrm{V}_{\text {gas }}$ and $\left(\mathrm{V}_{\text {rigid }}-\mathrm{V}_{\text {sixfold }}\right) / \mathrm{V}_{\text {gas, }}$ respectively, where $V_{\text {rigid }}$ and $V_{\text {normal }}$ represent the molar volume of $\mathrm{SiO}_{2}$ glass in noble gas media and non-gas media conditions, accordingly; $\mathrm{V}_{\text {gas }}$ - the molar volume of noble gas; $\mathrm{V}_{\text {sixfold }}$ - the molar volume of six-fold-coordinated $\mathrm{SiO}_{2}$ glass (Sato et al., 2011). We do not have an accurate determination of volume of silica in $\mathrm{Ne}$, instead we assume the volume change under pressure is same as the He case, as similar volume curves were suggested by integration method (Fig. S-3).

Basalt glass is compositionally more complex than silica glass with the addition of other cations. These cations can be classified into two categories: the network formers like $\mathrm{Ti}, \mathrm{Al}$ and network modifiers like $\mathrm{Mg}, \mathrm{Ca}, \mathrm{Na}$ and $\mathrm{K}$. The two sets of cations have distinct effects on gas solubility. Network modifiers tend to form bonds between the bridging $\mathrm{SiO}_{4}$ tetrahedra and lower the volume of void space. Their negative correlation with noble gas solubility has been experimentally observed (Tournour and Shelby, 2008a,b). On the other hand, network formers, which reside in the Si site, seem to have less of an influence on gas solubility.

\section{Geochemical Implications}

The geometrical packing and coordination of atoms in silicate melts and glasses are similar at ambient and high pressure conditions based on experimental observations (Williams and Jeanloz, 1988; Morard et al., 2020). Hence, the void space distribution in melt structure is likely to be comparable and our results here support significant solubility of helium and neon in high pressure silicate melts (Fig. 3). Furthermore, the partition coefficients between minerals and melts for noble gases are in the order of $10^{-3}$ (Karato, 2016). It is expected silicate melts should be an important host for noble gases. He and $\mathrm{Ne}$ are the $2^{\text {nd }}$ and $5^{\text {th }}$ most abundant elements in the solar system (Palme et al., 2014). For ${ }^{3} \mathrm{He} /{ }^{4} \mathrm{He}$ and ${ }^{20,21} \mathrm{Ne} /{ }^{22} \mathrm{Ne}$ isotopic ratios, the discrepancy of upper mantle material value from the atmospheric value has been a hot topic in geochemistry (e.g., Bekaert et al., 2019; Mukhopadhyay and Parai, 2019). Most answers to this question require a deep primordial reservoir which has unique geochemical features and survives mantle convection for the last 4.5 billion years. It has been noticed that some patches of partially molten rock might exist at the core mantle boundary (e.g., Wen et al. 2001). These melts may be able to host large amounts of noble gases like helium and neon with primordial and less radiogenic features. Other than the core-mantle boundary, partial melting may also occur at the top of the lower mantle due to dehydration melting (Fu et al., 2019). These layers might be perturbed by mantle convection more often and host noble gases with more radiogenic features. Therefore, the observed difference in noble gases ratios in OIBs and MORBs could be possibly due to sampling different melt reservoirs for noble gases. The storage of helium or neon discussed here reaches conditions beyond the range of this experiment, and due to the complex coordination environment change of silicon at higher pressures (Wang et al., 2014), and the high temperature conditions in deep Earth, directly applying our results to these conditions may not be suitable. However, the mechanism revealed in this study and previous studies (Clark et al., 2016; Sato et al., 2011; Weigel et al., 2012) (i.e. availability of interstitial void to compressed noble gases) is still valid and future structure simulation and void space analysis of heated silica/basalt glass at high pressures is needed. Geodynamic simulations are also needed to better estimate the degree of mixing during these processes.

Noble gas-silicate interaction may also have important implications for the composition of the atmospheres of other planetary bodies like Jupiter. It is found that the abundances of helium and neon in Jupiter's atmosphere are significantly lower than other noble gases, when compared to solar composition (Fortney, 2010). Our results suggest that this discrepancy could be related to interior processes in the planet, which account for the 'missing' helium and neon. Forming a He-Nesilicate composite at Jupiter's rocky core could be a viable option to lower the fraction of $\mathrm{He}$ and $\mathrm{Ne}$ in its atmosphere. Whether such a mechanism could explain the deficit of neon and helium in Jupiter's atmosphere requires further experimental and computational work. Our study demonstrates the controlling factors for noble gas solubility in a silicate melt are the noble gas size compared to void size, indicating that data on the structure of silicate melts with natural compositions at higher $P$ - $T$ is crucially needed in order to estimate the storage capacity of noble gases in deep planetary interiors

\section{Acknowledgements}

We appreciate constructive comments from two anonymous reviewers. AEG and WM acknowledge support from NSF Geophysics (EAR0738873). RJ acknowledges support from UC CMEC. Work was performed at GeoSoilEnviroCARS which is supported by NSF (EAR - 1634415) and DOE (DE-FG02-94ER14466). Use of the COMPRES-GSECARS gas loading system was supported by COMPRES under NSF (EAR -1606856) and GSECARS. The Advanced Photon Source is operated for the DOE Office of Science by Argonne National Laboratory (DE-AC02-06CH11357).

Editor: Anat Shahar

\section{Additional Information}

Supplementary Information accompanies this letter at https:// www.geochemicalperspectivesletters.org/article2105.

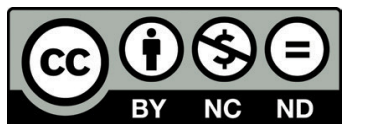

(C) 2021 The Authors. This work is distributed under the Creative Commons Attribution NonCommercial No-Derivatives 4.0 License, which permits unrestricted distribution provided the original author and source are credited. The material may not be adapted (remixed, transformed or built upon) or used for commercial purposes without written permission from the 
author. Additional information is available at https://www. geochemicalperspectivesletters.org/copyright-and-permissions.

Cite this letter as: Yang, H., Gleason, A.E., Tkachev, S.N., Chen, B., Jeanloz, R., Mao, W.L. (2021) Noble gas incorporation into silicate glasses: implications for planetary volatile storage. Geochem. Persp. Let. 17, 1-5.

\section{References}

Bajgain, S., GHosh, D.B., Karki, B.B. (2015) Structure and density of basaltic melts at mantle conditions from first-principles simulations. Nature Communications 6, 1-7.

BeKaert, D.V., Broadley, M.W., Caracausi, A., Marty, B. (2019) Novel insights into the degassing history of Earth's mantle from high precision noble gas analysis of magmatic gas. Earth and Planetary Science Letters 525, 115766.

Clark, A.N., Lesher, C.E., JACOBSEN, S.D., WANG, Y. (2016) Anomalous density and elastic properties of basalt at high pressure: Reevaluating of the effect of melt fraction on seismic velocity in the Earth's crust and upper mantle. Journal of Geophysical Research: Solid Earth 121, 4232-4248.

Coasne, B., Weigel, C., Polian, A., Kint, M., Rouquette, J., Haines, J., Foret M., VAcheR, R., Rufflé, B. (2014) Poroelastic Theory Applied to the Adsorption-Induced Deformation of Vitreous Silica. The Journal of Physical Chemistry B 118, 14519-14525.

Dewaele, A., Datchi, F., Loubeyre, P., Mezouar, M. (2008) High pressure-high temperature equations of state of neon and diamond. Physical Review B 77, 094106.

Fortney, J. (2010) Peering into Jupiter. Physics 3, 26

Fu, S., Yang, J., Karato, S., Vasiliev, A., Presniakov, M. Yu., GavrilliuK, A.G. Ivanova, A.G., Hauri, E.H., Okuchi, T., Purevjav, N., Lin, J. (2019) Water Concentration in Single-Crystal (Al,Fe)-Bearing Bridgmanite Grown From the Hydrous Melt: Implications for Dehydration Melting at the Topmost Lower Mantle. Geophysical Research Letters 46, 10346-10357.

Karato, S. (2016) Physical basis of trace element partitioning: A review. American Mineralogist 101, 2577-2593

Klotz, S., Chervin, J.C., Munsch, P., Lemarchand, G. (2009) Hydrostatic limits of 11 pressure transmitting media. Journal of Physics D Applied Physics 42,075413 .

LIU, J., LIN, J.-F. (2014) Abnormal acoustic wave velocities in basaltic and (Fe,Al)bearing silicate glasses at high pressures. Geophysical Research Letters 41 8832-8839.

Loubeyre, P., LeToullec, R., Pinceaux, J.P., MaO, H.K., Hu, J., Hemley, R.J. (1993) Equation of state and phase diagram of solid $4 \mathrm{He}$ from single-crysta x-ray diffraction over a large P-T domain. Physical Review Letters 71 2272-2275.

Malavasi, G., Menziani, M.C., Pedone, A., Segre, U. (2006) Void size distribution in MD-modelled silica glass structures. Journal of Non-Crystalline Solids 352 285-296.

Morard, G., Hernandez, J.-A., Guarguaglini, M., Bolis, R., Benuzzi-Mounaix, A., Vinci, T., Figuet, G., Baron, M.A., Shim, S.H., Ko, B., Gleason, A.E., MaO, W.L., Alonso-Mori, R., Lee, H.J., Nagler, B., Galtier, E., Sokaras, D., Glenzer, S.H., Andrault, D., Garbarino, G., Mezouar, M., SCHUStER, A.K., RAVASIO, A. (2020) In situ X-ray diffraction of silicate liquids and glasses under dynamic and static compression to megabar pressures. Proceedings of the National Academy of Sciences 117, 11981-11986.

Mukhopadhyay, S., Parai, R. (2019) Noble Gases: A Record of Earth's Evolution and Mantle Dynamics. Annual Review of Earth and Planetary Sciences 47, 389-419.

Palme, H., Lodders, K., Jones, A. (2014) 2.2 - Solar System Abundances of the Elements. In: Holland, H.D., TuREKIAN, K.K. (Eds.) Treatise on Geochemistry. Second Edition, Elsevier, Oxford, 15-36, doi: 10.1016 B978-0-08-095975-7.00118-2.

Polian, A., Grimsditch, M. (1993) Sound velocities and refractive index of densified $\alpha-\mathrm{SiO}_{2}$ to $25 \mathrm{GPa}$. Physical Review B 47, 13979-13982.

ReID, R.C., Prausnitz, J.M., Poling, B.E. (1987) The properties of gases and liquids. McGraw Hill Book Co., New York, NY

Rosa, A.D., BouhifD, M.A., Morard, G., Briggs, R., Garbarino, G., IrIFune, T., Mathon, O., Pascarelli, S. (2020) Krypton storage capacity of the Earth's lower mantle. Earth and Planetary Science Letters 532, 116032.

Ross, M., MaO, H.K., Bell, P.M., Xu, J.A. (1986) The equation of state of dense argon: A comparison of shock and static studies. The Journal of Chemical Physics 85, 1028-1033.
SAKAmaki, T., Kono, Y., WANG, Y., PARK, C., YU, T., Jing, Z., SHen, G. (2014) Contrasting sound velocity and intermediate-range structural order between polymerized and depolymerized silicate glasses under pressure. Earth and Planetary Science Letters 391, 288-295.

SANCheZ-VAlLE, C., Bass, J.D. (2010) Elasticity and pressure-induced structural changes in vitreous $\mathrm{MgSiO} 3$-enstatite to lower mantle pressures. Earth and Planetary Science Letters 295, 523-530.

Sanloup, C., Schmidt, B.C., Perez, E.M.C., Jambon, A., Gregoryanz, E., Mezouar, M. (2005) Retention of Xenon in Quartz and Earth's Missing Xenon. Science. American Association for the Advancement of Science 310, 1174-1177.

Sato, T., Funamori, N., Yagi, T. (2011) Helium penetrates into silica glass and reduces its compressibility. Nature Communications 2, 345.

SHACKELFORD, J.F., MASARYK, J.S. (1978) The interstitial structure of vitreous silica. Journal of Non-Crystalline Solids 30, 127-134.

Shen, G., Mei, Q., Prakapenka, V.B., Lazor, P., Sinogeikin, S., Meng, Y., Park, C. (2011) Effect of helium on structure and compression behavior of $\mathrm{SiO} 2$ glass. Proceedings of the National Academy of Sciences 108, 6004-6007.

Tournour, C.C., Shelby, J.E. (2008a) Neon solubility in silicate glasses and melts. Physics and Chemistry of Glasses 49, 8.

Tournour, C.C., ShelBy, J.E. (2008b) Helium solubility in alkali silicate glasses and melts. Physics and Chemistry of Glasses - European Journal of Glass Science and Technology Part B 49, 207-215.

Wang, Y., Sakamaki, T., Skinner, L.B., Jing, Z., Yu, T., Kono, Y., Park, C., Shen, G., RIVERS, M.L., SutTON, S.R. (2014) Atomistic insight into viscosity and density of silicate melts under pressure. Nature Communications 5, 3241

Weigel, C., Polian, A., Kint, M., Rufflé, B., Foret, M., Vacher, R. (2012) Vitreous Silica Distends in Helium Gas: Acoustic Versus Static Compressibilities. Physical Review Letters 109, 245504.

Wen, L., Silver, P.G., James, D.E., KueHnel, R. (2001) Seismic evidence for a thermochemical boundary at the base of the Earth's mantle. Earth and Planetary Science Letters 189, 141-153.

Williams, Q., Jeanloz, R. (1988) Spectroscopic Evidence for Pressure-Induced Coordination Changes in Silicate Glasses and Melts. Science 239, 902-905.

Yoshimura, Y., Stewart, S.T., Somayazulu, M., MaO, H., Hemley, R.J. (2006) Highpressure $\mathrm{x}$-ray diffraction and Raman spectroscopy of ice VIII. The Journal of Chemical Physics 124, 024502.

Zha, C., Hemley, R.J., MaO, H., Duffy, T.S., Meade, C. (1994) Acoustic velocities and refractive index of $\mathrm{SiO} 2$ glass to $57.5 \mathrm{GPa}$ by Brillouin scattering. Physical Review B 50, 13105-13112.

ZHANG, C., DuAn, Z., LI, M. (2010) Interstitial voids in silica melts and implication for argon solubility under high pressures. Geochimica et Cosmochimica Acta 74, $4140-4149$ 